\title{
Disconjugacy and disfocality criteria for second order singular half-linear differential equations
}

\author{
by Ondřej Došlý and Alexander Lomtatidze (Brno)
}

\begin{abstract}
We establish Vallée Poussin type disconjugacy and disfocality criteria for the half-linear second order differential equation

$$
u^{\prime \prime}=p(t)|u|^{\alpha}\left|u^{\prime}\right|^{1-\alpha} \operatorname{sgn} u+g(t) u^{\prime},
$$

where $\alpha \in(0,1]$ and the functions $p, g \in L_{\text {loc }}(a, b)$ are allowed to have singularities at the end points $t=a, t=b$ of the interval under consideration.
\end{abstract}

1. Introduction. In this paper we investigate oscillatory properties and solvability of two-point BVP's associated with the half-linear second order differential equation

$$
u^{\prime \prime}=p(t)|u|^{\alpha}\left|u^{\prime}\right|^{1-\alpha} \operatorname{sgn} u+g(t) u^{\prime},
$$

where $\alpha \in(0,1],-\infty<a<b<\infty$, and the functions $p, g \in L_{\mathrm{loc}}(a, b)$ are allowed to have singularities at the end points $t=a, t=b$. Multiplying (1.1) by $\alpha\left|u^{\prime}\right|^{1-\alpha}$ we get the half-linear equation in the form more frequently considered in the literature:

$$
\left(\Phi\left(u^{\prime}\right)\right)^{\prime}=\alpha p(t) \Phi(u)+\alpha g(t) \Phi\left(u^{\prime}\right), \quad \Phi(s):=|s|^{\alpha} \operatorname{sgn} s .
$$

However, if $\alpha \neq 1$, equation (1.1) is of a slightly different kind than (1.2) and the solution sets of (1.1) and (1.2) are not identical. Indeed, suppose that $a \leq t_{1}<t_{2}<t_{3}<t_{4} \leq b$ and that there exist solutions $u, v$ of (1.1) such that $u\left(t_{1}\right)=0=u^{\prime}\left(t_{2}\right), v^{\prime}\left(t_{3}\right)=0=v\left(t_{4}\right)$; moreover, since the solution space of (1.1) is homogeneous, we can suppose that $u\left(t_{2}\right)=v\left(t_{3}\right)$. Now, the

1991 Mathematics Subject Classification: Primary 34C10.

Key words and phrases: half-linear equation, disconjugacy, disfocality, Vallée Poussin type criterion.

Supported by the Grant A1019902 of the Grant Agency of Academy of Sciences and by the Grant 201/99/0295 of the Czech Grant Agency. 
function

$$
\widetilde{u}(t)= \begin{cases}u(t), & t_{1} \leq t \leq t_{2} \\ u\left(t_{2}\right), & t_{2} \leq t \leq t_{3} \\ v(t), & t_{3} \leq t \leq t_{4}\end{cases}
$$

is a solution of (1.1) but not of (1.2). This means, in particular, that the Sturmian separation theorem (which holds for (1.2)) does not extend to (1.1), i.e., (1.1) in general admits coexistence of a nonzero solution with a solution having two or more zeros in an interval under consideration.

Oscillation theory of (1.1) and (1.2) attracted a considerable attention in the recent years, see e.g. $[2,5-7,12-16]$ and the references given therein. It was shown that oscillatory properties of (1.1), (1.2) are similar to those of the linear equation

$$
u^{\prime \prime}=p(t) u+g(t) u^{\prime}
$$

and methods like variational and Riccati technique, which are typical in linear oscillation theory, extend with minor modifications also to half-linear equations.

In this paper we prefer to write half-linear equations in the form (1.1) since then the equation can be regarded as a particular case of the nonlinear second order equation $u^{\prime \prime}=f\left(t, u, u^{\prime}\right)$. The assumption $\alpha \in(0,1]$ is enforced by the fact that for $\alpha>1$ the right-hand side of (1.1) becomes singular in the phase variable $u^{\prime}$.

In 1929 de la Vallée Poussin [21] proved that the second order linear equation (1.3) (with $g, p$ continuous on $[a, b]$ ) is disconjugate in the interval $[a, b]$, provided

$$
b-a<2 \int_{0}^{\infty} \frac{d t}{1+A t+B t^{2}}, \quad A=\max _{t \in[a, b]}|g(t)|, \quad B=\max _{t \in[a, b]}|p(t)|,
$$

and as a consequence of (1.4) he showed that two consecutive zeros $a, b$ of a nontrivial solution of (1.3) have to satisfy

$$
2 A(b-a)+\frac{1}{2} B(b-a)^{2}>1 .
$$

The last condition (given in the monograph by Sansone [20] as the "real" Vallée Poussin criterion) has been extended in various directions (see [9, $19,22]$ and also the survey paper [1] containing a comprehensive bibliography). Finally, because of "misinterpretation" of the original result of Vallée Poussin (1.4), this condition was rediscovered by Cohn in [3]. Note also that an interesting treatment of Vallée Poussin's condition (1.4) can be found in the paper by Opial [19].

Our main result, Theorem 3.1, concerns a Vallée Poussin's type criterion for (1.1), where the coefficients $g, p$ are allowed to be singular at the end 
points of the interval under consideration. Moreover, in contrast to the previous criteria of this kind, we only use one-sided estimates of the functions $g, p$.

The paper is organized as follows. In the next section we formulate some auxiliary results concerning unique solvability of (1.1) with singular end points $a, b$ and disconjugacy of this equation in terms of solvability of a certain second order differential inequality. Section 3 is devoted to the main results of the paper: Vallée Poussin type disconjugacy and disfocality criteria for (1.1). The last section contains some remarks concerning these statements.

2. Auxiliary results. Throughout the paper we use the following notation: $\widetilde{C}_{\text {loc }}^{1}(a, b)$ denotes the set of functions $u:(a, b) \rightarrow \mathbb{R}$ such that $u, u^{\prime}$ are absolutely continuous on each compact subinterval of $(a, b)$. Next, $L(a, b)$ is the usual space of Lebesgue integrable functions on $(a, b)$.

By a solution of equation (1.1) we understand a function $u \in \widetilde{C}_{\mathrm{loc}}^{1}(a, b)$ which satisfies (1.1) almost everywhere in $(a, b)$. It is easy to see that if $u$ is a solution of (1.1) then $\lambda u$ is a solution as well. The fact that the solution space of (1.1) has just one half of linearity (homogeneity but not additivity) justifies the terminology "half-linear equation" for (1.1).

Observe that if we define

$$
r(t)=\exp \left\{\int_{(a+b) / 2}^{t} g(s) d s\right\}
$$

then (1.1) can be written in the form

$$
\left(\frac{u^{\prime}}{r(t)}\right)^{\prime}=\frac{p(t)}{r(t)}|u|^{\alpha}\left|u^{\prime}\right|^{1-\alpha} \operatorname{sgn} u .
$$

In this paper we investigate the problem of the existence of a nontrivial solution of (1.1) satisfying the boundary conditions

$$
\begin{aligned}
& u\left(a_{1}+\right)=0=u\left(b_{1}-\right), \\
& u\left(a_{1}+\right)=0=u^{\prime}\left(b_{1}-\right)
\end{aligned}
$$

for some $a_{1}, b_{1} \in[a, b], a_{1}<b_{1}$. In our investigation we do not exclude the case when both functions $p, q$ have singularities at $a$ and/or $b$. In particular, we will investigate problem (1.1), (2.2) under the assumptions

$$
r(t) \in L(a, b), \quad p(t)\left(\frac{1}{r(t)} \int_{a}^{t} r(s) d s \int_{t}^{b} r(s) d s\right)^{\alpha} \in L(a, b)
$$


and problem (1.1), (2.3) with

$$
r(t) \in L(a, b), \quad p(t)\left(\frac{\int_{a}^{t} r(s) d s}{r(t)}\right)^{\alpha} \in L(a, b) .
$$

Notice that (2.4) holds e.g. if

$$
|p(t)| \leq \frac{C}{[(t-a)(b-t)]^{1+\varepsilon}}, \quad|g(t)| \leq C+\delta\left[\frac{1}{t-a}+\frac{1}{b-t}\right]
$$

and condition (2.5) holds if

$$
|p(t)| \leq \frac{C}{(t-a)^{1+\varepsilon}}, \quad|g(t)| \leq C+\frac{\delta}{t-a},
$$

where $C>0, \varepsilon \in(0, \alpha), \delta \in[0,1)$. Note also that condition (2.4) guarantees that each solution $u$ of (1.1) has finite (one-sided) limits at the end points $a, b$, and condition (2.5) implies that $\lim _{t \rightarrow b-} u^{\prime}(t)$ exists and it is finite (see [2], Lemma 2.2).

Now we show that if the integrability condition (2.4) holds then the zero initial conditions determine the trivial solution of (1.1).

Lemma 2.1. Suppose that (2.4) holds and $a_{1} \in[a, b)$ (resp. $\left.b_{1} \in(a, b]\right)$. Then the trivial solution $u \equiv 0$ is the only solution of (1.1) satisfying the condition

or respectively,

$$
u\left(a_{1}+\right)=0, \quad \liminf _{t \rightarrow a_{1}+}\left|\frac{u^{\prime}(t)}{r^{\alpha}(t)}\right|=0
$$

$$
u\left(b_{1}-\right)=0, \quad \liminf _{t \rightarrow b_{1}-}\left|\frac{u^{\prime}(t)}{r^{\alpha}(t)}\right|=0 .
$$

Pro of. See [2, Lemma 2.8].

LEMma 2.2. Suppose that (2.4) holds and $u$ is a nontrivial solution of (1.1) satisfying $u(a+)=0$ (resp. $(u(b-)=0)$. Then for any continuously differentiable bounded function $v:(a, b) \rightarrow \mathbb{R}$ we have

$$
\liminf _{t \rightarrow a+}\left|\frac{v^{\prime}(t) u(t)}{u^{\prime}(t)}\right|=0, \quad \text { or respectively, } \quad \liminf _{t \rightarrow b-}\left|\frac{v^{\prime}(t) u(t)}{u^{\prime}(t)}\right|=0 .
$$

P r o of. Suppose, by contradiction, that the statement fails to hold. Then by Lemma 2.1 there exist $\varepsilon>0$ and $a_{0} \in(a, b)$ such that

$$
|u(t)| \neq 0 \quad \text { and } \quad\left|v^{\prime}(t)\right|>\varepsilon\left|\frac{u^{\prime}(t)}{u(t)}\right| \quad \text { for } a<t<a_{0} .
$$

Hence

$$
|v(t)|>\int_{t}^{a_{0}}\left|v^{\prime}(s)\right| d s-\left|v\left(a_{0}\right)\right|>\varepsilon \ln \frac{u\left(a_{0}\right)}{u(t)}-\left|v\left(a_{0}\right)\right|
$$

for $a<t<a_{0}$, which contradicts the boundedness of $v$. 
Now we turn our attention to disconjugacy and disfocality criteria for (1.1).

Definition. Equation (1.1) is said to be disconjugate in the interval $[a, b]$ if it does not have a nontrivial solution satisfying $u\left(a_{1}+\right)=0=u\left(b_{1}-\right)$ for any $a_{1}, b_{1} \in[a, b], a_{1}<b_{1}$, and it is said to be left (right) disfocal in $[a, b]$ if it does not possess a nontrivial solution satisfying $u\left(a_{1}+\right)=0=u^{\prime}\left(b_{1}+\right)$ $\left(u^{\prime}\left(a_{1}+\right)=0=u\left(b_{1}-\right)\right)$ for any $a_{1}, b_{1} \in[a, b], a_{1}<b_{1}$.

LEMma 2.3. Let (2.4) hold and suppose that there exist $c \in[a, b]$ and a bounded positive function $v \in \widetilde{C}_{\mathrm{loc}}^{1}(a, b)$ with finite limits $v(a+)>0$, $v(b-)>0$ such that $v^{\prime}(c)=0$ and

$$
\begin{aligned}
v^{\prime \prime} \leq p(t)|v(t)|^{\alpha}\left|v^{\prime}(t)\right|^{1-\alpha}+g(t) v^{\prime}(t) & \text { for } a<t<b, \\
v^{\prime}(t)(c-t)>0 & \text { for } a<t<b, t \neq c .
\end{aligned}
$$

Then (1.1) is disconjugate in $[a, b]$.

Proof. We will prove the statement in case $c \in(a, b)$. When $c=a$ or $c=b$ we proceed in a similar way. Suppose, by contradiction, that $u$ is a nontrivial solution of (1.1) having at least two zeros in $[a, b]$, i.e., there exist $a_{1} \in[a, b)$ and $b_{1} \in(a, b]$ such that $u\left(a_{1}+\right)=0=u\left(b_{1}-\right)$. Then there exist $t_{1}, t_{2} \in\left(a_{1}, b_{1}\right), t_{1} \leq t_{2}$, such that $u^{\prime}\left(t_{1}\right)=0, u^{\prime}\left(t_{2}\right)=0$ and

$$
u^{\prime}(t)>0 \quad \text { for } a_{1}<t<t_{1}, \quad u^{\prime}(t)<0 \quad \text { for } t_{2}<t<b_{1} .
$$

Define the functions $\varrho_{1}$ and $\varrho_{2}$ by

$$
\varrho_{1}(t)=\left|\frac{u^{\prime}(t)}{u(t) r(t)}\right|^{\alpha}, \quad \varrho_{2}(t)=\left|\frac{v^{\prime}(t)}{v(t) r(t)}\right|^{\alpha} .
$$

By a direct computation one can verify that

$$
\begin{aligned}
& \varrho_{1}^{\prime}(t)=\alpha\left(\frac{p(t)}{r^{\alpha}(t)}-r(t) \varrho_{1}^{(\alpha+1) / \alpha}(t)\right) \operatorname{sgn} \frac{u^{\prime}(t)}{u(t)}, \quad t \in\left(a_{1}, t_{1}\right) \cup\left(t_{2}, b_{1}\right), \\
& \varrho_{2}^{\prime}(t) \leq \alpha\left(\frac{p(t)}{r^{\alpha}(t)}-r(t) \varrho_{2}^{(\alpha+1) / \alpha}(t)\right) \operatorname{sgn} v^{\prime}(t), \quad a_{1}<t<b_{1} .
\end{aligned}
$$

In addition, by Lemmas 2.1 and 2.2 we have

$$
\begin{gathered}
\varrho_{1}\left(a_{1}+\right)=\infty, \quad \varrho_{1}\left(b_{1}-\right)=\infty, \\
\liminf _{t \rightarrow a_{1}+} \frac{\varrho_{2}(t)}{\varrho_{1}(t)}=0=\liminf _{t \rightarrow b_{1}-} \frac{\varrho_{2}(t)}{\varrho_{1}(t)} .
\end{gathered}
$$

Suppose first that $t_{1}<c$. Then by $(2.8)$ there exist $\tau_{1} \in\left(a_{1}, t_{1}\right)$ and $\varepsilon \in$ $\left(0, t_{1}-\tau_{1}\right)$ such that

$$
\varrho_{1}(t)<\varrho_{2}(t) \quad \text { for } \tau_{1}<t<\tau_{1}+\varepsilon, \quad \varrho_{1}\left(\tau_{1}\right)=\varrho_{2}\left(\tau_{1}\right) .
$$


If we take into account (2.9), then (2.7) implies

$$
\begin{aligned}
\varrho_{1}(t) & =\varrho_{1}\left(\tau_{1}\right)+\int_{\tau_{1}}^{t} \frac{p(s)}{r^{\alpha}(s)} d s-\int_{\tau_{1}}^{t} r(s) \varrho_{1}^{(\alpha+1) / \alpha}(s) d s \\
& >\varrho_{2}\left(\tau_{1}\right)+\int_{\tau_{1}}^{t} \frac{p(s)}{r^{\alpha}(s)} d s-\int_{\tau_{1}}^{t} r(s) \varrho_{2}^{(\alpha+1) / \alpha}(s) d s \geq \varrho_{2}(t)
\end{aligned}
$$

for $\tau_{1}<t<\tau_{1}+\varepsilon$, which contradicts (2.9), hence $t_{1} \geq c$.

Now suppose that $t_{1}=c$. By the same argument as above there exist $c_{1} \in(a, c]$ and $\varepsilon \in\left(0, c_{1}-a_{1}\right)$ such that

$$
\varrho_{2}(t)<\varrho_{1}(t) \text { for } c_{1}-\varepsilon<t<c_{1}, \quad \varrho_{1}\left(c_{1}\right)=\varrho_{2}\left(c_{1}\right) .
$$

Set $w(t)=\varrho_{1}(t)-\varrho_{2}(t)$ and

$$
\varphi(x, y)= \begin{cases}\frac{1}{|x|-|y|}\left(|x|^{(\alpha+1) / \alpha}-|y|^{(\alpha+1) / \alpha}\right) & \text { for }|x| \neq|y|, \\ 0 & \text { for }|x|=|y| .\end{cases}
$$

Then

$$
w^{\prime}(t) \geq-\alpha r(t) w(t) \varphi\left(\varrho_{1}(t), \varrho_{2}(t)\right) \quad \text { for } c_{1}-\varepsilon<t<c_{1} .
$$

Inequality (2.11) implies that for $t \in\left(c_{1}-\varepsilon, c_{1}\right)$,

$$
w(t) \leq w\left(c_{1}\right) \exp \left[\alpha \int_{t}^{c_{1}} r(s) \varphi\left(\varrho_{1}(s), \varrho_{2}(s)\right) d s\right] .
$$

Since $w\left(c_{1}\right)=0$, the last inequality contradicts (2.10). Consequently, we have proved that $t_{1}>c$. In the same way one can prove that $t_{2}<c$, but this impossible, since $t_{1} \leq t_{2}$.

REMARK 2.1. The statement of Lemma 2.3 remains valid if $c \in(a, b)$, $v \in \widetilde{C}_{\mathrm{loc}}^{1}(a, c) \cup \widetilde{C}_{\mathrm{loc}}^{1}(c, b)$ and $v^{\prime}(c-) \geq 0 \geq v^{\prime}(c+)$.

In the same way as in Lemma 2.3 one can prove the following disfocality criterion for (1.1).

LEMmA 2.4. Suppose that there exists a bounded function $v \in \widetilde{C}_{\mathrm{loc}}^{1}(a, b)$ with finite limits $v(a+)>0, v^{\prime}(b-)>0, v^{\prime}(t)>0$ on $(a, b)$, and satisfying the inequality

$$
v^{\prime \prime}(t) \leq p(t) v^{\alpha}(t)\left(v^{\prime}(t)\right)^{1-\alpha}+g(t) v(t), \quad a<t<b .
$$

Then the boundary value problem (1.1), (2.3) has only the trivial solution for every $a_{1}, b_{1} \in[a, b]$.

REMARK 2.2. In the same way as in Lemma 2.4 we can prove that the boundary value problem (1.1), $u^{\prime}(a+)=0, u(b-)=0$ has no nontrivial 
solution provided there exists a bounded function $v \in \widetilde{C}_{\text {loc }}^{1}(a, b)$ such that $v^{\prime}(a+)<0, v(b-)>0, v^{\prime}(t)<0$ and (2.12) holds.

3. Main results. The main results of our paper, the Vallée Poussin type disconjugacy and disfocality criteria for (1.1), read as follows.

Theorem 3.1. Suppose that (2.4) holds, there exist $c \in[a, b], l_{i j} \geq 0$, $i, j=1,2$, and differentiable functions $\beta, \gamma$ such that $\beta(t)>0$ for $t \in(a, c)$, $\gamma(t)>0$ for $t \in(c, b)$,

$$
\beta^{-1 / \alpha} \in L(a, c), \quad \gamma^{-1 / \alpha} \in L(c, b)
$$

and

$$
\begin{aligned}
& \int_{0}^{\infty} \frac{d s}{l_{11}+l_{12} s+s^{(\alpha+1) / \alpha}}>\alpha \int_{a}^{c} \beta^{-1 / \alpha}(s) d s, \\
& \int_{0}^{\infty} \frac{d s}{l_{21}+l_{22} s+s^{(\alpha+1) / \alpha}}>\alpha \int_{c}^{b} \gamma^{-1 / \alpha}(s) d s,
\end{aligned}
$$

where

$$
\begin{aligned}
& \beta^{(\alpha+1) / \alpha}(t) p(t) \geq-l_{11}, \quad \beta^{1 / \alpha}(t)\left[g(t)+\frac{\beta^{\prime}(t)}{\alpha \beta(t)}\right] \geq-l_{12}, a<t<c, \\
& \gamma^{(\alpha+1) / \alpha}(t) p(t) \geq-l_{21}, \quad \gamma^{1 / \alpha}(t)\left[g(t)+\frac{\gamma^{\prime}(t)}{\alpha \gamma(t)}\right] \leq l_{22}, \quad c<t<b .
\end{aligned}
$$

Then the BVP (1.1), (2.2) has only the trivial solution for any $a \leq a_{1}<$ $b_{1} \leq b$, i.e., (1.1) is disconjugate in $[a, b]$.

Proof. We prove the statement in case $c \in(a, b)$, the cases $c=a$ or $c=b$ may treated in the same way. Without loss of generality we can suppose that both $l_{11} \neq 0, l_{21} \neq 0$. Let $\gamma_{1}>0$ and $\gamma_{2}>0$ be such that

$$
\begin{aligned}
& \int_{0}^{\gamma_{1}} \frac{d s}{l_{11}+l_{12} s+s^{(\alpha+1) / \alpha}}=\alpha \int_{a}^{c} \beta^{-1 / \alpha}(s) d s, \\
& \int_{0}^{\gamma_{2}} \frac{d s}{l_{21}+l_{22} s+s^{(\alpha+1) / \alpha}}=\alpha \int_{c}^{b} \gamma^{-1 / \alpha}(s) d s .
\end{aligned}
$$

Define the functions $\varrho_{1}, \varrho_{2}$ (implicitly) by the equalities

$$
\begin{array}{ll}
\int_{\varrho_{1}(t)}^{\gamma_{1}} \frac{d s}{l_{11}+l_{12} s+s^{(\alpha+1) / \alpha}}=\alpha \int_{a}^{t} \beta^{-1 / \alpha}(s) d s, & a<t \leq c, \\
\int_{\varrho_{2}(t)}^{\gamma_{2}} \frac{d s}{l_{21}+l_{22}+s^{(\alpha+1) / \alpha}}=\alpha \int_{t}^{b} \gamma^{-1 / \alpha}(s) d s, & c \leq t<b .
\end{array}
$$


Obviously, $\varrho_{1}(t)>0$ for $a<t<c, \varrho_{1}(a+)=\gamma_{1}, \varrho_{1}(c)=0, \varrho_{2}(t)>0$ for $c<t<b, \varrho_{2}(b-)=\gamma_{2}, \varrho_{2}(c)=0$. Let

$$
v(t)= \begin{cases}\exp \left[-\int_{t}^{c} \beta^{-1 / \alpha}(s) \varrho_{1}^{1 / \alpha}(s) d s\right] & \text { for } a<t \leq c \\ \exp \left[-\int_{c}^{t} \gamma^{-1 / \alpha}(s) \varrho_{2}^{1 / \alpha}(s) d s\right] & \text { for } c<t<b\end{cases}
$$

Then

$$
\begin{gathered}
v(t)>0 \quad \text { for } a<t<b, \\
v^{\prime}(t)(c-t)>0 \quad \text { for } t \in(a, c) \cup(c, b), \quad v^{\prime}(c)=0,
\end{gathered}
$$

there exist finite limits $v(a+)>0, v(b-)>0$ and we have, for $t \in(a, c)$,

$$
\begin{aligned}
v^{\prime}(t) & =\left(\frac{\varrho_{1}(t)}{\beta(t)}\right)^{1 / \alpha} v(t), \\
v^{\prime \prime}(t) & =v(t)\left(\frac{\varrho_{1}(t)}{\beta(t)}\right)^{2 / \alpha}+v(t) \frac{1}{\alpha}\left(\frac{\varrho_{1}(t)}{\beta(t)}\right)^{1 / \alpha-1}\left(\frac{\varrho_{1}(t)}{\beta(t)}\right)^{\prime} .
\end{aligned}
$$

Consequently,

$v^{\prime \prime}(t)-p(t) v^{\alpha}\left|v^{\prime}(t)\right|^{1-\alpha}-g(t) v^{\prime}(t)$

$=\frac{1}{\alpha}\left(\frac{\varrho_{1}(t)}{\beta(t)}\right)^{1 / \alpha-1} v(t)\left[\left(\frac{\varrho_{1}(t)}{\beta(t)}\right)^{\prime}+\alpha\left(\frac{\varrho_{1}(t)}{\beta(t)}\right)^{(\alpha+1) / \alpha}-\alpha p(t)-\alpha g(t) \frac{\varrho_{1}(t)}{\beta(t)}\right]$.

The estimates (3.2) now imply that

$$
v^{\prime \prime}(t) \leq p(t)|v(t)|^{\alpha}\left|v^{\prime}(t)\right|^{1-\alpha}+g(t) v^{\prime}(t), \quad a<t<c ;
$$

the same inequality holds also for $c<t<b$ as can be verified by a direct computation. Consequently, by Lemma 2.3 every solution of (1.1) has at most one zero in $[a, b]$.

REMARK 3.1. A closer examination of the proof of the previous theorem reveals that this statement is actually a "double disfocality criterion". Indeed, $\left(3.1_{1}\right),\left(3.2_{1}\right)$ imply that (1.1) is left disfocal in $[a, c]$ and $\left(3.1_{2}\right),\left(3.2_{2}\right)$ imply right disfocality in $[c, b]$. This, in turn, implies disconjugacy of (1.1) in $[a, b]$.

This observation immediately suggests the following (left) disfocality criterion.

THEOREM 3.2. Suppose that (2.5) holds and there exists a differentiable function $\beta$ satisfying $\beta(t)>0$ for $t \in(a, b), \beta^{-1 / \alpha} \in L(a, b)$, such that

$$
\int_{0}^{\infty} \frac{d t}{l_{11}+l_{12} s+s^{(\alpha+1) / \alpha}}>\alpha \int_{a}^{b} \beta^{-1 / \alpha}(s) d s,
$$


where

$$
\beta^{(\alpha+1) / \alpha}(t) p(t) \geq-l_{11}, \quad \beta^{1 / \alpha}(t)\left[g(t)+\frac{\beta^{\prime}(t)}{\beta(t)}\right] \geq-l_{12}, \quad a<t<b .
$$

Then the BVP (1.1), (2.3) has only the trivial solution, i.e., (1.1) is left disfocal in $[a, b]$.

Typical examples of functions $\beta, \gamma$ which meet the assumptions of Theorem 3.1 are specified in the following corollary.

Corollary 3.1. Let $\lambda_{1}, \lambda_{2} \in[0, \alpha), c \in[a, b], l_{i j} \geq 0, i, j=1,2$, and

$$
\begin{aligned}
& \int_{0}^{\infty} \frac{d t}{l_{11}+l_{12} s+s^{(\alpha+1) / \alpha}}>\frac{\alpha^{2}}{\alpha-\lambda_{1}}(c-a)^{\left(\alpha-\lambda_{1}\right) / \alpha}, \\
& \int_{0}^{\infty} \frac{d t}{l_{21}+l_{22} s+s^{(\alpha+1) / \alpha}}>\frac{\alpha^{2}}{\alpha-\lambda_{2}}(b-c)^{\left(\alpha-\lambda_{2}\right) / \alpha},
\end{aligned}
$$

where

$$
\begin{aligned}
& (t-a)^{\lambda_{1}(\alpha+1) / \alpha} p(t) \geq-l_{11}, \quad(t-a)^{\lambda_{1} / \alpha}\left[g(t)+\frac{\lambda_{1}}{\alpha(t-a)}\right] \geq-l_{12}, \quad a<t<c, \\
& (b-t)^{\lambda_{2}(\alpha+1) / \alpha} p(t) \geq-l_{21}, \quad(b-t)^{\lambda_{2} / \alpha}\left[g(t)-\frac{\lambda_{2}}{\alpha(b-t)}\right] \leq l_{22}, \quad c<t<b .
\end{aligned}
$$

Then (1.1) is disconjugate in $[a, b]$.

Proof. Substitute $\beta(t)=(t-a)^{\lambda_{1}}, \gamma(t)=(b-t)^{\lambda_{2}}$ in Theorem 3.1.

4. Remarks and comments. In this final section we give some remarks concerning the results of the previous section.

(i) The Vallée Poussin criterion (1.4) is a special case of Theorem 3.1. Indeed, it suffices to substitute $c=(b+a) / 2, \beta \equiv 1 \equiv \gamma, \alpha=1$ and take into account that

$$
\int_{0}^{\infty} \frac{d t}{1+M t+N t^{2}}=\int_{0}^{\infty} \frac{d t}{N+M t+t^{2}} .
$$

Theorem 3.1 also extends the results of [10], Theorem 4.4, and [11], Lemma 1.7, to half-linear equations.

(ii) Harris [8] proved that (1.3) is disconjugate in $[a, b]$ if there exists a differentiable function $\mu$ such that (1.4) holds, where $A, B$ are given by

$$
A=\max _{t \in[a, b]}|g(t)+2 \mu(t)|, \quad B=\max _{t \in[a, b]}\left|p(t)-g(t) \mu(t)-\mu^{2}(t)+\mu^{\prime}(t)\right| .
$$

Substituting $\mu \equiv 0$ we get (1.4). The criterion of Harris is essentially based on the Riccati substitution $\varrho=y^{\prime} / y+\mu$ whereas our criterion from Theo- 
rem 3.1 is based on the substitution

$$
\varrho(t)=\beta(t)\left(\frac{y^{\prime}(t)}{y(t)}\right)^{\alpha} .
$$

This suggests investigating disconjugacy of (1.1) using the combined substitution

$$
\varrho(t)=\beta(t)\left(\frac{y^{\prime}(t)}{y(t)}\right)^{\alpha}+\mu(t) .
$$

We have tried to follow this idea, but till now we have obtained no result analogous to those of Harris.

(iii) The strict inequalities in (3.1) cannot be replaced by equalities as shown by the following example. Let $c \in(a, b), \lambda_{1}, \lambda_{2}$ and $l_{12}, i, j=1,2$, be the same as in Corollary 3.1 and

$$
\begin{aligned}
& \int_{0}^{\infty} \frac{d s}{l_{11}+l_{12} s+s^{(\alpha+1) / \alpha}}=\frac{\alpha^{2}}{\alpha-\lambda_{1}}(c-a)^{\left(\alpha-\lambda_{1}\right) / \alpha}, \\
& \int_{0}^{\infty} \frac{d s}{l_{21}+l_{22} s+s^{(\alpha+1) / \alpha}}=\frac{\alpha_{2}}{\alpha-\lambda_{2}}(b-c)^{\left(\alpha-\lambda_{2}\right) / \alpha} .
\end{aligned}
$$

Define the functions $\varrho_{1}, \varrho_{2}$ by

$$
\begin{aligned}
& \int_{\varrho_{1}(t)}^{\infty} \frac{d s}{l_{11}+l_{12} s+s^{(\alpha+1) / \alpha}}=\frac{\alpha^{2}}{\alpha-\lambda_{1}}(c-a)^{\left(\alpha-\lambda_{1}\right) / \alpha}, \quad a<t \leq c, \\
& \int_{\varrho_{2}(t)}^{\infty} \frac{d s}{l_{21}+l_{22}+s^{(\alpha+1) / \alpha}}=\frac{\alpha_{2}}{\alpha-\lambda_{2}}(b-c)^{\left(\alpha-\lambda_{2}\right) / \alpha}, \quad c \leq t<b .
\end{aligned}
$$

Then

$$
\begin{gathered}
\varrho_{1}(a+)=\infty, \quad \varrho_{2}(b-)=\infty, \quad \varrho_{1}(c)=0, \quad \varrho_{2}(c)=0, \\
\varrho_{1}(t)>0 \quad \text { for } a<t<c, \quad \varrho_{2}(t)>0 \quad \text { for } c<t<b .
\end{gathered}
$$

Now it is not difficult to verify that there exists $\varepsilon>0$ (sufficiently small) and $\delta \in(0,1)$ such that

$$
\begin{array}{ll}
\frac{\alpha^{2}}{\alpha-\lambda_{1}}(t-a)^{\left(\alpha-\lambda_{1}\right) / \alpha}>\delta \int_{\varrho_{1}(t)}^{\infty} \frac{d s}{s^{(\alpha+1) / \alpha}}=\alpha \delta \varrho_{1}^{-1 / \alpha}(t) & \text { for } a<t<a+\varepsilon, \\
\frac{\alpha^{2}}{\alpha-\lambda_{2}}(b-t)^{\left(\alpha-\lambda_{2}\right) / \alpha}>\alpha \delta \varrho_{2}^{-1 / \alpha}(t) & \text { for } b-\varepsilon<t<b .
\end{array}
$$

Consequently,

$$
\lim _{t \rightarrow a+} \int_{t}^{c}(s-a)^{-\lambda_{1} / \alpha} \varrho_{1}^{1 / \alpha}(s) d s=\infty=\lim _{t \rightarrow b-} \int_{c}^{t}(b-s)^{-\lambda_{2} / \alpha} \varrho_{2}^{1 / \alpha}(s) d s .
$$


One can verify directly that the function $v$ given by (3.3) (with $\beta(t)=$ $\left.(t-a)^{\lambda_{1}}, \gamma(t)=(b-t)^{\lambda_{2}}\right)$ is a solution of (1.1) with

$$
\begin{aligned}
& \left.\begin{array}{l}
p(t)=-l_{11}(t-a)^{-\lambda_{1}(1+\alpha) / \alpha} \\
g(t)=\left(-l_{12}-\frac{\lambda_{1}}{\alpha(t-a)}\right)^{-\lambda_{1} / \alpha} \\
p(t)=-l_{21}(b-t)^{-\lambda_{2}(\alpha+1) / \alpha} \quad \text { for } a<t<c, \\
g(t)=\left(l_{22}+\frac{\lambda_{2}}{\alpha(b-t)}\right)^{-\lambda_{2} / \alpha}
\end{array}\right\} \quad \text { for } c<t<b .
\end{aligned}
$$

On the other hand, by (4.1), $v(a+)=0, v(b-)=0$, hence (1.1) is not disconjugate in $[a, b]$.

\section{References}

[1] J. Andres, On the criterion of de la Vallée Poussin, Publ. Math. Debrecen 45 (1994), 145-152.

[2] T. Chantladze, N. Kandelaki and A. Lomtatidze, On zeros of solutions of second order singular half-linear equations, Mem. Differential Equations Math. Phys. 17 (1999), 127-154.

[3] J. H. E. Cohn, On an oscillation criterion of de la Vallée Poussin, Quart. J. Math. Oxford Ser. (2) 39 (1988), 173-174.

[4] O. Došlý, A remark on conjugacy of half-linear second order differential equations, Math. Slovaca, to appear.

[5] -, Oscillation criteria for half-linear second order differential equations, Hiroshima Math. J. 28 (1998), 507-521.

[6] A. Elbert and T. Kusano, Principal solutions of nonoscillatory half-linear differential equations, Adv. Math. Sci. Appl. 18 (1998), 745-759.

[7] A. Elbert, T. Kusano and T. Tanigawa, An oscillatory half-linear differential equation, Arch. Math. (Brno) 33 (1997), 165-172.

[8] B. J. Harris, On an oscillation criterion of Cohn, Quart. J. Math. Oxford Ser. (2) 42 (1991), 309-313.

[9] P. Hartman and A. Wintner, On an oscillation criterion of de la Vallée Poussin, Quart. Appl. Math. 13 (1955), 330-332.

[10] I. T. Kiguradze and A. G. Lomtatidze, On certain boundary value problems for second order linear ordinary differential equations with singularities, J. Math. Anal. Appl. 101 (1984), 325-347.

[11] I. T. Kiguradze and B. L. Shekhter, Singular boundary value problems for second order ordinary differential equations, Itogi Nauki i Tekh. Ser. Sovrem. Probl. Mat. 30 (1987), 105-201 (in Russian); English transl.: J. Soviet Math. 43 (1988), 2340-2417.

[12] T. Kusano and M. Naito, Oscillation and nonoscillation criteria for second order quasilinear differential equations, Acta Math. Hungar. 76 (1997), 81-99.

[13] T. Kusano, Y. Naito and A. Otaga, Strong oscillation and nonoscillation of quasilinear differential equations of second order, Differential Equations Dynam. Systems 2 (1994), 1-10. 
[14] H. J. Li, Oscillation criteria for half-linear second order differential equations, Hiroshima Math. J. 25 (1995), 571-583 .

[15] H. J. Li and C. C. Yeh, Sturmian comparison theorem for half-linear second order differential equations, Proc. Roy. Soc. Edinburgh 125A (1996), 1193-1204.

[16] A. Lomtatidze, Oscillation and nonoscillation of Emden-Fowler type equation of second order, Arch. Math. (Brno) 32 (1996), 181-193.

[17] J. D. Mirzov, On some analogs of Sturm's and Kneser's theorems for nonlinear systems, J. Math. Anal. Appl. 53 (1976), 418-425.

[18] - Asymptotic Properties of Nonlinear Nonautonomous Systems of Ordinary Differential Equations, Adygeja, Majkop, 1994.

[19] Z. Opial, Sur une inégalité de Ch. de la Vallée Poussin dans la théorie de l'équation différentielle linéaire du second ordre, Ann. Polon. Math. 6 (1959), 81-91.

[20] G. Sansone, Equazioni differenziali nel campo reale, parte prima, Zanichelli, Bologna, 1948.

[21] Ch. de la Vallée Poussin, Sur l'équation différentielle linéaire du second ordre. Détermination d'une intégrale par deux valeurs assignés. Extension aux équations d'ordre n, J. Math. Pures Appl. 8 (1929), 125-144.

[22] D. Willet, Generalized de la Vallée Poussin disconjugacy test for linear differential equations, Canad. Math. Bull. 14 (1971), 419-428.

Ondřej Došlý

Mathematical Institute

Czech Academy of Sciences

Žižkova 22

CZ-616 62 Brno, Czech Republic

E-mail: dosly@math.muni.cz
Alexander Lomtatidze

Department of Mathematics Masaryk University Janáčkovo nám. 2a CZ-662 95 Brno, Czech Republic E-mail: bacho@math.muni.cz

Reçu par la Rédaction le 12.5.1999 